\title{
NOUVELLE
}

\section{Abaisser \\ la pression artérielle permet de limiter la microangiopathie cérébrale}

\author{
Christophe Tzourio, Carole Dufouil, Nathalie Tzourio-Mazoyer
}

\author{
C. Tzourio, C. Dufouil : \\ Inserm U.708, Université \\ Pierre et Marie Curie, \\ Hôpital Pitié-Salpêtrière, \\ 47, boulevard de l'Hôpital, \\ 75013 Paris, France. \\ tzourio@chups.jussieu.fr \\ N. Tzourio-Mazoyer : \\ GIN UMR 6194 CNRS-CEA- \\ Université Caen-Paris 5, \\ Centre Cycéron, \\ boulevard Henri Becquerel, \\ 14074 Caen Cedex, France.
}

était possible de ralentir leur évolution. Le principal facteur de risque modifiable des HSB étant, selon de nombreuses études, I'hypertension artérielle, il était logique de faire I'hypothèse selon laquelle un abaissement des chiffres de pression artérielle permettrait de limiter leur évolution. C'est pour tester cette hypothèse que nous avons implémenté sieurs centaines ou plusieurs milliers de sujets [2-5] ont permis de montrer que les HSB avaient un profil de risque qui les rapprochent des accidents vasculaires cérébraux, avec comme principaux facteurs de risque l'âge et I'hypertension artérielle. Le mécanisme généralement évoqué pour expliquer la survenue des HSB est celui d'une artériolosclérose, une dégénérescence de la paroi des petites artères perforantes irriguant la substance blanche cérébrale. Cette microangiopathie cérébrale a pour conséquence une souffrance ischémique de la zone irriguée dans laquelle peuvent se voir une démyélinisation, une gliose, et parfois un infarcissement.

Plusieurs études ont montré que, contrairement aux premières impressions, tiels sur leurs mécanismes et leurs con-

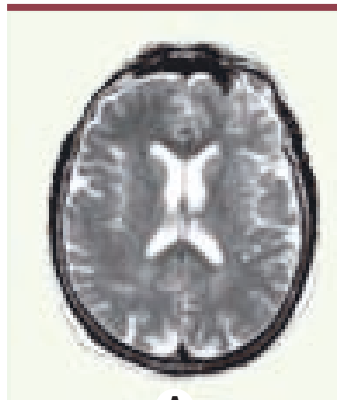

A

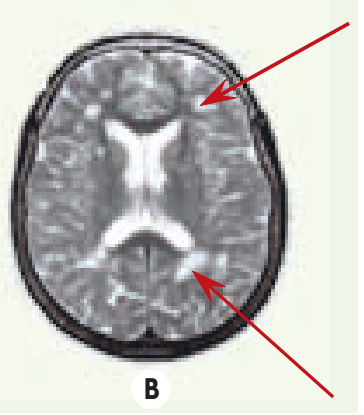

Figure 1. IRM cérébrale, coupes pondérées T2. Coupes de même niveau chez deux sujets, $\boldsymbol{A}$ et $\boldsymbol{B}$. Chez le sujet $\boldsymbol{A}$ qui était normotendu, I'IRM ne met pas en évidence d'hypersignaux. Le sujet $B$ qui avait une HTA présente de nombreux hypersignaux de la substance blanche dans la substance blanche profonde et en regard des cornes ventriculaires (flèches). les HSB ne sont en réalité pas anodins et que leur accumulation est associée à un risque augmenté de diverses pathologies graves : accidents vasculaires cérébraux ( $A V C)$, détérioration cognitive, voire démence, troubles de la marche, dépression [6-8]. Malgré cette accumulation de preuves impliquant les HSB dans ce qui peut être considéré comme un vieillissement accéléré du cerveau, il n'avait jusquelà jamais été montré qu'il une étude sur l'IRM cérébrale dans un grand essai thérapeutique international, l'étude PROGRESS, portant sur plus de 6000 patients et démontrant l'intérêt d'un traitement abaissant la tension artérielle après un premier accident vasculaire cérébral [9]. Parallèlement à la réalisation de cet essai, nous avons mis en place une sous-étude baptisée «IRM-PROGRESS » dans 10 Centres français [10]. Dans cette étude de suivi par IRM cérébrale, nous avons réalisé pour chacun des 192 patients inclus dans ces centres, deux IRM cérébrales à 3 ans d'intervalle selon des procédures d'acquisition homogènes. Ces IRM étaient ensuite transférées au GIN (Groupe d'imagerie neurofonctionnelle) du centre Cycéron de Caen (France), spécialisé dans l'analyse d'image. Par des techniques complexes de traitement d'image, les deux examens réalisés au début et à la fin de l'étude étaient rendus aussi comparables que possible, afin de détecter et de mesurer de façon très précise les HSB apparus lors du suivi. Pour chaque sujet, le volume total des HSB détectés entre le $1^{\text {er }}$ et le $2^{\mathrm{e}}$ examen IRM était évalué. Ce volume a ensuite été comparé chez les sujets recevant le traitement actif et chez ceux recevant le placebo.

Entre la première et la seconde IRM, la baisse de pression artérielle était en moyenne de 11,2 $\mathrm{mmHg}$ pour la pression systolique et $4,3 \mathrm{~mm} \mathrm{Hg}$ pour la pression 
diastolique dans le groupe traité par rapport au groupe placebo. Durant le suivi, 24 patients $(12,5 \%)$ développèrent des nouveaux HSB. Par rapport au groupe placebo, le groupe traité avait une diminution de $43 \%$ du risque de nouveaux HSB (intervalle de confiance à $95 \%,-7 \%$ à $89 \% ; p=0,17$ ) et un volume moyen total de nouveaux HSB plus faible $\left(0,4 \pm 0,8\right.$ versus $2,0 \pm 0,7 \mathrm{~mm}^{3}$; $p=0,012$ ). Cette différence était nettement plus marquée pour le groupe des patients ayant un grade sévère d'HSB sur la première $\operatorname{IRM}(0,0 \pm 0,0$ versus $7,6 \pm 1,0 ; p<0,0001)$, c'est-à-dire les plus exposés au risque de déclin cognitif et de démence.

Ces données confirment, pour la première fois, l'intuition des médecins et chercheurs: l'abaissement de la pression artérielle permet bien de diminuer le risque de lésions ischémiques cérébrales. Mais surtout, elles suggèrent que l'effet du traitement associant un inhibiteur de l'enzyme de conversion et un diurétique est particulièrement puissant puisque le volume des nouvelles lésions était 5 fois moins important chez les patients recevant le traitement actif que chez ceux sous placebo.
Ces résultats offrent des perspectives importantes en matière de prévention des troubles cognitifs liés à l'âge et aux facteurs vasculaires. Ils soulèvent de nouvelles questions: existe-t-il une sous-population de patients ayant une forte évolutivité de leur grade en HSB? La prévention des HSB s'accompagne-t-elle d'une réduction du risque de démence? L'effet préventif d'un traitement abaissant la pression artérielle s'observe-il dans des populations plus larges, comme par exemple chez les hypertendus n'ayant pas eu d'AVC? D'autres traitements peuvent-ils également ralentir l'évolution des HSB ? Les réponses à ces questions nécessitent de mettre en place des études de grande taille comprenant la réalisation d'IRM cérébrales, donc onéreuses. Mais la question de la prévention des complications neurologiques de I'HTA, en particulier la prévention des démences, est un des futurs grands défis dans ce domaine. Il ne pourra être relevé que par une collaboration étroite entre épidémiologistes, cliniciens et spécialistes de l'imagerie cérébrale, comme dans l'étude IRM-PROGRESS. $\diamond$

Blood pressure lowering and limitation of the cerebral microangiopathy

\section{RÉFÉRENCES}

1. Kertesz A, Black SE, Tokar G, et al. Periventricular and subcortical hyperintensities on magnetic resonance imaging. Rims, caps and unidentified bright objects. Arch Neurol 1988 ; $45: 404-8$.

2. Longstreth WT, Manolio TA, Arnold A, et al. Clinical correlates of white matter findings on cranial magnetic resonance imaging of 3301 elderly people : the cardiovascular health study. Stroke $1996 ; 27: 1274-82$.

3. Bots ML, van Swieten JC, Breteler M, et al. Cerebral white matter lesions and atherosclerosis in the Rotterdam study. Lancet 1993 ; 341 : 1232-7.

4. Liao DP, Cooper L, Cai JW, et al. Presence and severity of cerebral white matter lesions and hypertension, its treatment, and its control : the ARIC study. Stroke 1996 ; $27: 2262-70$.

5. Dufouil C, de Kersaint Gilly A, Besancon V, et al. Longitudinal study of blood pressure and white matter hyperintensities : the EVA MRI cohort. Neurology 2001 ; $56: 921-6$

6. Van Gijn J. White matters : small vessels and slow thinking in old age. Lancet $2000 ; 356: 612-3$.

7. Dufouil C, Alperovitch A, Tzourio C. Influence of education on the relationship between white matter lesions and cognition. Neurology $2003 ; 60: 831-6$.

8. Vermeer SE, Prins ND, den Heijer T, et al. Silent brain infarcts and the risk of dementia and cognitive decline. N EnglJ Med 2003 ; 348 : 1215-22.

9. PROGRESS Collaborative Group. Randomised trial of a perindopril-based blood-pressure-lowering regimen among 6105 individuals with previous stroke or transient ischaemic attack. Lancet $2001 ; 358: 1033-41$.

10. Dufouil C, Chalmers J, Coskun 0, et al. Effects of blood pressure lowering on cerebral white matter hyperintensities in patients with stroke : the PROGRESS (Perindopril protection against recurrent stroke study) magnetic resonance imaging substudy. Circulation 2005; $112: 1644-50$.

\section{NOUVELLE}

\section{Neuro-anatomie du traitement de l'espace}

Michel Thiebaut de Schotten, Paolo Bartolomeo

\author{
Inserm U.610, Pavillon Claude Bernard, \\ Hôpital Pitié-Salpêtrière, 47, boulevard de l'Hôpital, \\ 75013 Paris, France. \\ paolo.bartolomeo@chups.jussieu.fr
}

> Les lésions de l'hémisphère droit provoquent souvent une «négligence spatiale gauche », trouble qui gêne la rééducation et qui a pour conséquence une réduction de l'autonomie et une réhabilitation sociale compromise.

L'étude de la négligence spatiale gauche est un élément important à la fois sur le plan clinique et aussi afin de mieux comprendre les mécanismes cérébraux du traitement de l'espace.
La localisation anatomique de ce trouble est restée controversée, les uns impliquant le lobule pariétal inférieur droit, les autres la partie antérieure du gyrus temporal supérieur.

La stimulation électrique peropératoire au cours d'intervention chirurgicale sur I'hémisphère droit a été utilisée pour explorer les fonctions visuo-spatiales. La stimulation du lobule pariétal inférieur et de la partie postérieure - et non pas la partie antérieure du gyrus temporal supérieur - provoque une déviation vers la droite de la section médiane d'une ligne, mais la réponse la plus nette se produit lors d'une stimulation sous-corticale. Grâce à une nouvelle technique d'IRM, le tracking de fibres, nous avons pu montrer que la région stimulée correspondait à une voie pariéto-frontale, appelée le fasciculus occipito-frontal supérieur. Ainsi, il apparaît que la communication pariéto- 